\title{
Limitations of atom-centered dielectric functions in implicit solvent models
}

\author{
Jessica M.J. Swanson, John Mongan, J. Andrew McCammon \\ 20th May 2005
}

Four additional potentials of mean force (PMF) are included here to supplement the data presented in the main article.

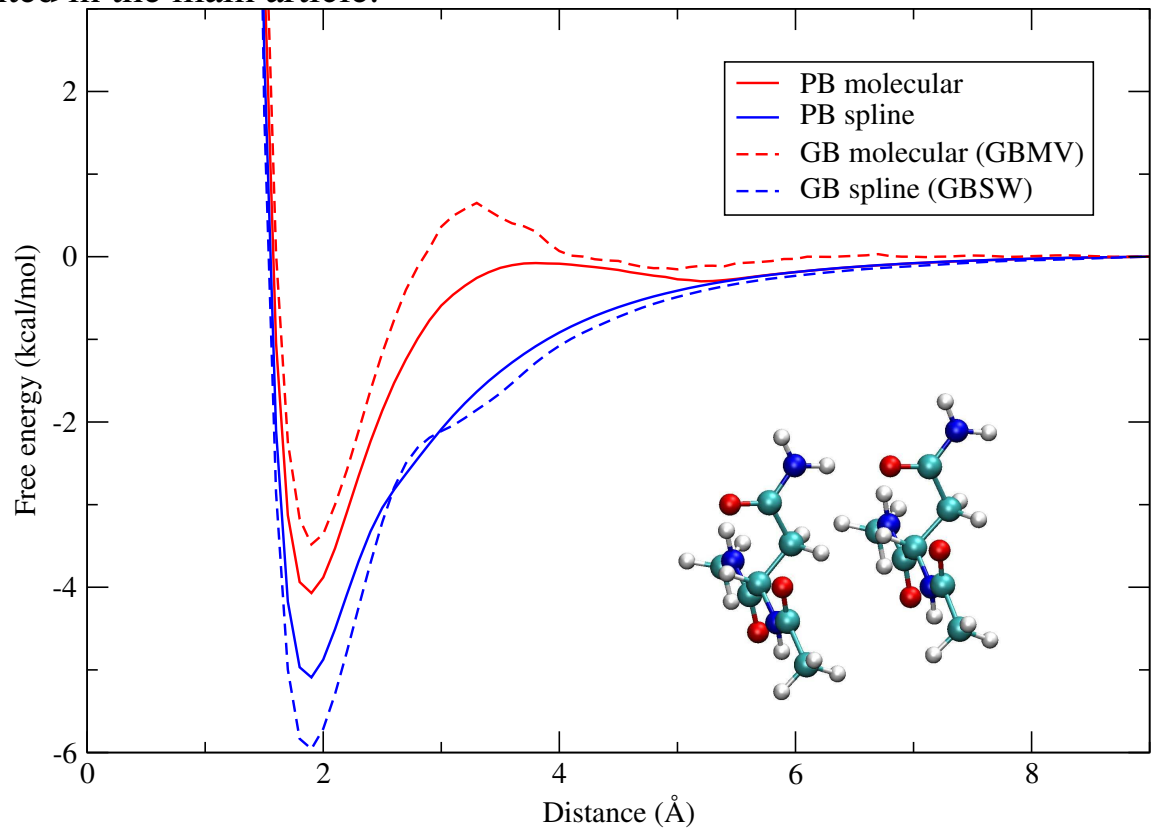

Figure 1: PMF for the illustrated asparagine-asparagine hydrogen bond. Distances are measured between the hydrogen and oxygen atoms participating in the bond. 


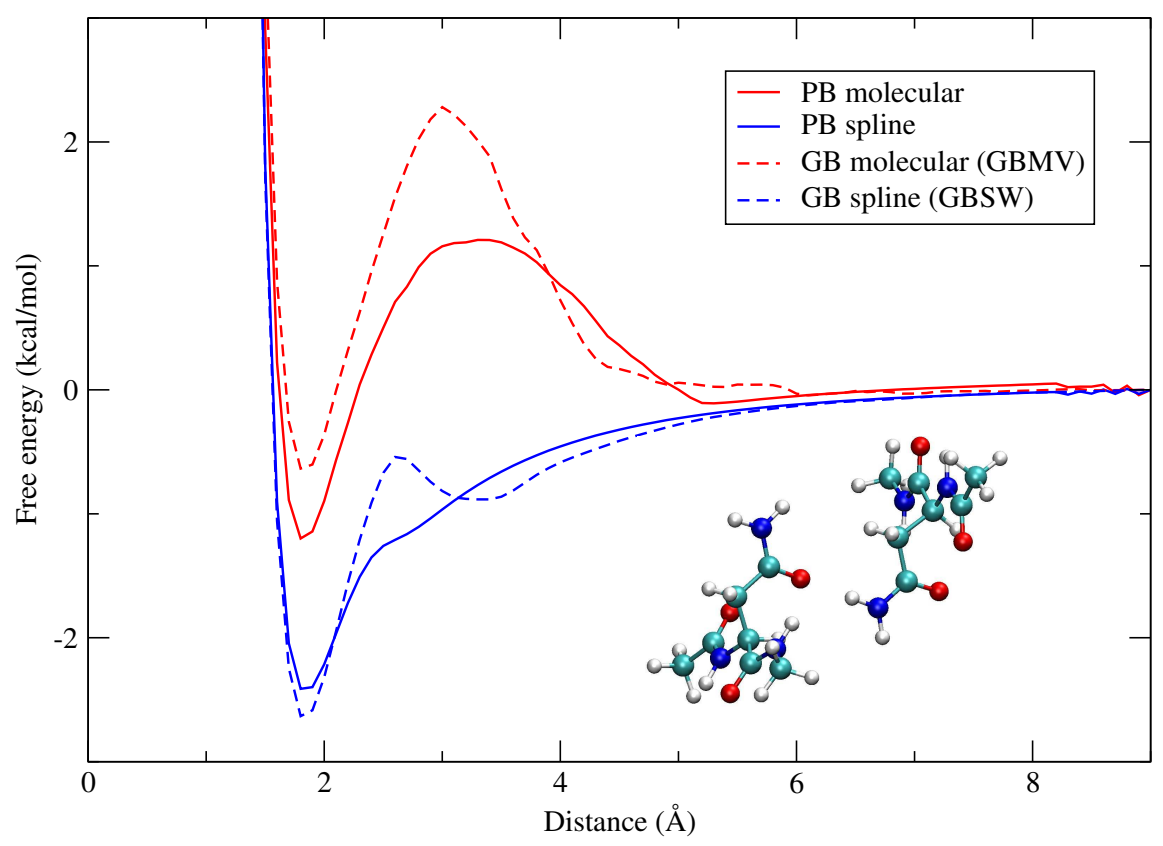

Figure 2: PMF for the illustrated asparagine-asparagine hydrogen bond, which is similar to that seen in Figure 1, but with one of the bonding partners rotated 180 degrees about the axis of the hydrogen bond. Distances are measured between the hydrogen and oxygen atoms participating in the bond.

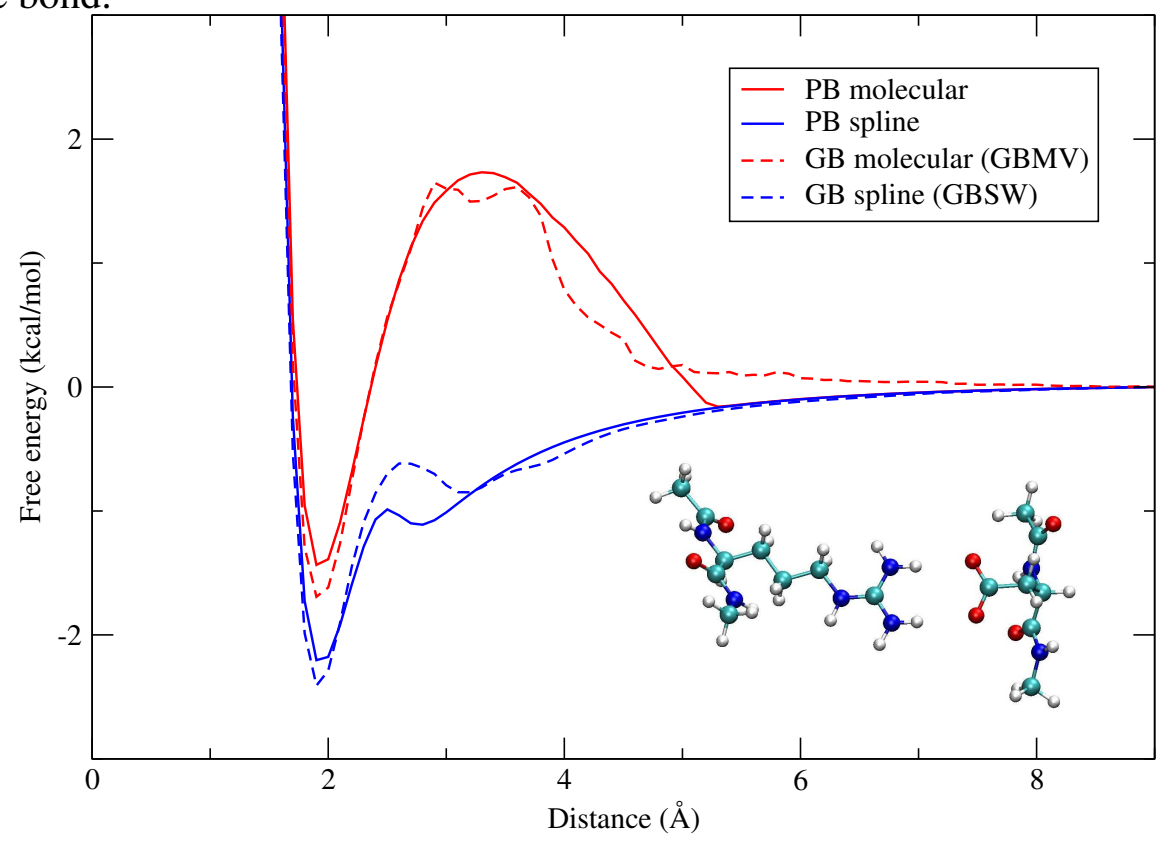

Figure 3: PMF for the illustrated arginine-aspartate salt bridge. Distances are measured between the hydrogen and oxygen atoms participating in the bond. 


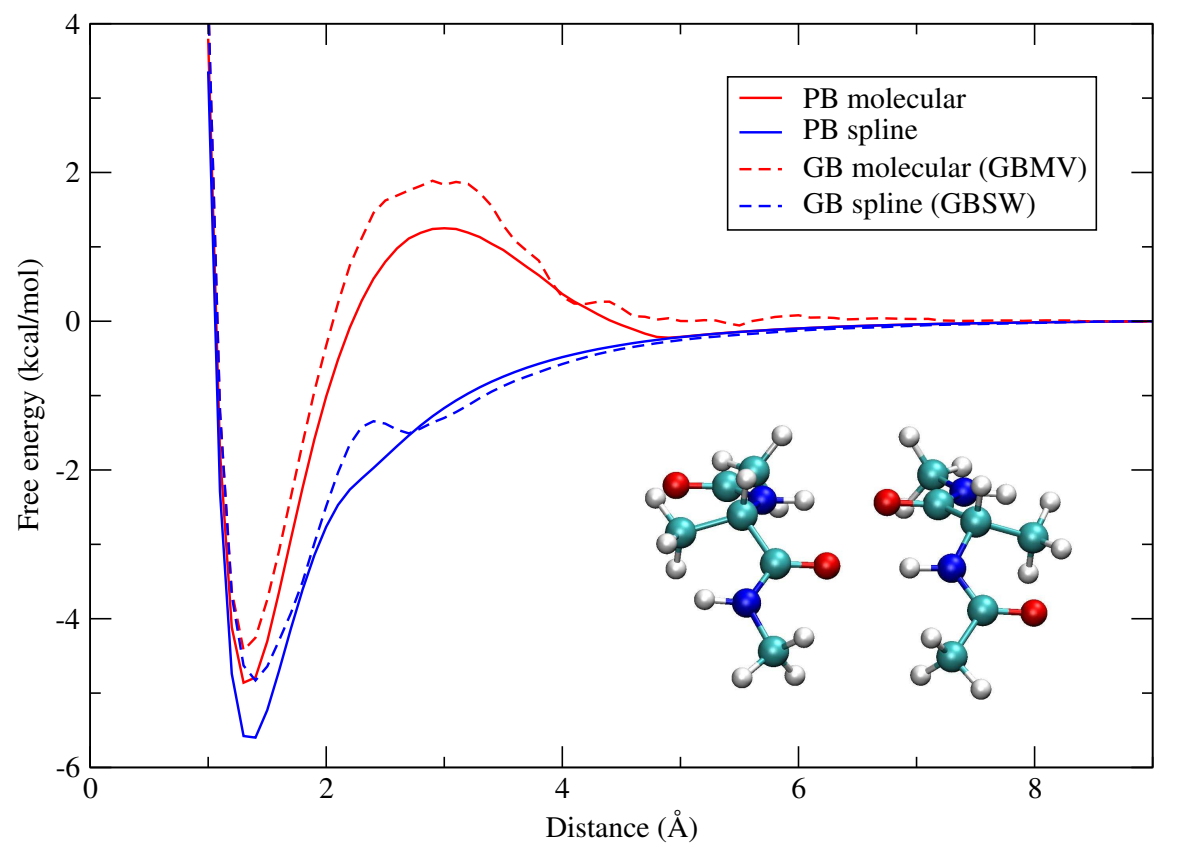

Figure 4: PMF for the hydrogen bonding between the backbones of two blocked alanine molecules. Distances are measured between the hydrogen and oxygen atoms participating in the bond. 